\title{
GENERATING NAVIGATION MODELS FROM EXISTING BUILDING DATA
}

\author{
L. Liu, S. Zlatanova
}

GIS Technology, OTB-Research for the Built Environment, Faculty of Architecture and the built Environment, delft University of Technology, Jaffalaan 9, 2628BX, Delft, The Netherlands \{1.liu-1, s.zlatanova $\} @$ tudelft.nl

\author{
Commission IV, Working Group IV/7
}

KEY WORDS : Indoor Navigation, Navigation Model Generation, Building Data, Indoor Modelling

\begin{abstract}
:
Research on indoor navigation models mainly focuses on geometric and logical models .The models are enriched with specific semantic information which supports localisation, navigation and guidance. Geometric models provide information about the structural (physical) distribution of spaces in a building, while logical models indicate relationships (connectivity and adjacency) between the spaces. In many cases geometric models contain virtual subdivisions to identify smaller spaces which are of interest for navigation (e.g. reception area) or make use of different semantics. The geometric models are used as basis to automatically derive logical models. However, there is seldom reported research on how to automatically realize such geometric models from existing building data (as floor plans) or indoor standards (CityGML LOD4 or IFC).
\end{abstract}

In this paper, we present our experiments on automatic creation of logical models from floor plans and CityGML LOD4. For the creation we adopt the Indoor Spatial Navigation Model (INSM) which is specifically designed to support indoor navigation. The semantic concepts in INSM differ from daily used notations of indoor spaces such as rooms and corridors but they facilitate automatic creation of logical models.

\section{INTRODUCTION}

There is a lot of research on indoor space subdivision to support localisation, navigation (path finding) and guidance. Combinations of geometric, logical and semantic models are extensively investigated to support multi-purpose navigation (Lorenz et al., 2006; Stofel et al., 2007; Thill et al., 2011; Schaap et al., 2012). Geometric models contain details about spaces where agents (humans and robots) can move (walk, drive, or fly) or construction elements such as walls and columns acting as obstacles to interrupt agent's movement. Logical models provide information about topology of the spaces which can be expressed by adjacency, connectivity, etc. The link between logical and geometric models is usually straightforward: the nodes of logical models represent the spaces of geometric models and the edges of logical models represent topological relationships (Lee, 2004; Meijers et al., 2005; Becker et al., 2009). Semantic models provide the meaning of different building parts (spaces or construction elements). They are usually combined with geometric models although pure semantic models are reported as well (Yang and Worboys, 2011; Winter, 2012) .

The logical model can relatively easily be derived from geometric models when buildings are regularly structured. This is not the case when a building has complex shape, intermediate floors, semi-open spaces, irregular shapes, or non-horizontal floors $(\mathrm{Pu}$ and Zlatanova, 2005). Therefore a subdivision is required to be able to create as many nodes as needed for a logical model. Thus the generation of a logical model includes two steps:1) dividing indoor space into subspaces (which represent nodes), and 2) deriving the topological relationships between the subspaces (which represent edges). Automatic space subdivision is the main challenge in this process. Two approaches can be distinguished here: 1) semantic subdivision, which aims at identifying meaningful subspaces (such as coffee corner or reception) and 2) geometric subdivision, which decomposes the space according to a geometric criteria (grid, triangulation, Voronoi diagrams, etc.). The two approaches has controversies. If the automatic decomposition is ensured (i.e. by geometric approaches), the semantics is unclear; if the semantics of the sub-spaces is well-defined, it is difficult to provide automatic subdivision algorithm.

Our research is related to semantic subdivisions. Presently, there are several indoor models based on semantic subdivision, but no algorithms are provided to derive them from commonly available building data (e.g. floor plans, IFC or CityGML). This paper presents a method to generate logical model (i.e. connectivity network) from existing building geometry. The method is devised on the basis of an indoor space subdivision model named Indoor Navigation Space Model (INSM). INSM is a semantically rich model, which follows quite closely the original subdivision of a building but contains different naming of spaces. 
Such a specific vocabulary facilitates the construction of the connectivity network and path finding (Liu and Zlatanova, 2012).

This paper is organised as follows. Section 2 introduces related work; section 3 explains our generation method; section 4 presents test results and analyses them. Section 5 concludes the paper and addresses the importance of semantics for navigation.

\section{RELATED WORK}

Different logical modes of buildings can be yielded by the different subdivision methods. A subdivision is dependent on the type of application, i.e. which is the minimal space unit that needs to be identified. As mentioned above, the subspaces determine the nodes. But for one set of nodes, various relationships can be established. For example, a connectivity network provides relationships that indicates an agent can pass from one subspace to another. An adjacency network denotes all the neighbours of a given space. Furthermore, not all spaces might be considered (or accessible) in a specific navigation case, which will result in another type of logical model, i.e. accessibility network. Thus the logical model, which is a topological model, highly depends on subdivision result and the relationships between the subspaces.

At present, there is quite limited research on derivation of topological models from 3D models. The literature review shows that 2D geometry, such as floor plans, is frequently used to generate adjacency and connectivity network (Meijers et al., 2005; Lorenz et al., 2006; Stoffel et al., 2007). There are 3D approaches as well but they are either based on 2D floor plans (e.g. Tilll et al., 2011) or specific application (Schaap et al., 2008), or at quite conceptual level (Brown et al., 2013; Becker et al., 2009). In many approaches 3D model is mostly used for visualisation, after the path is constructed on 2D floor plan. However, some 3D building models, such as CityGML and Building Information Model (BIM), include many valuable geometric details and semantics of indoor elements, although their semantics on building information (e.g. floor, ceiling, wall, space $e t c$.) may need to be further refined for indoor navigation.

Semantics is a pivotal element of indoor navigation models. Semantics can be applied to facilitate the generation of topological model. For instance, it is relatively straightforward to obtain connectivity relations between indoor subspaces with knowledge of doors and rooms. Furthermore, navigation-related semantics of indoors (e.g. navigable space, obstacle, etc.) facilitates routing, and user-related semantics (rooms, office, corridor, etc) is more perceivable to users during navigation process. This type of semantics indicates the meaning and functions of indoor elements in terms of navigation and on the basis of geometry. Various semantic models have been developed with the focus of either users or easy extraction of logical network. For example the campus model of Richter $e$ al. is a typical example on user-related semantics. IndoorGML (a proposal for a standard) is an example that a model focuses on navigation network.
Indoor Navigation Space Model (INSM) is proposed to automate the generation of a logical model (Liu and Zlatanova, 2012). In INSM an indoor space is decomposed to different parts with explicit semantics (meaning) in terms of navigation. This is to say that connectivity network can be easily abstracted based on INSM. All the involved subspaces are assigned formal definitions and relations. INSM assumes that the whole indoor space is subdivided into non-overlapping semantic subspaces with distinct functions. Hence subspaces might not be necessarily strict to physical subdivisions. Basic subspaces of INSM are Navigable space cell (NSC), Opening $(O P N)$ and Obstacle $(O B S)$. NSC represents normally rooms, corridors, offices, etc. where an agent can be located; $O P N$ indicates open transition spaces from one NSC to another NSC for user's movement. Typical $O P N$ are doors; $O B S$ denotes the spaces which cannot be accessed by users. All the three kinds of subspaces correspond either to polygons (in 2D floor plans), or to $3 \mathrm{D}$ multiple surfaces (i.e. boundary representation) or to solid geometry.

The other semantic spaces are derivative to the three basic subspaces. Vertical Unit $(V U)$ is a NSC where agents can move in vertical directions (upward and downward); Horizontal Unit $(H U)$ is a NSC where agents can move in horizontal directions; End is a $H U$ which only connects one another NSC at most; Connector $(C O N)$ is a NSC which connects at least two distinct $N S C$; Vertical Connector $(V C)$ is a $H U$ which connects at least two other distinct $N S C$, meanwhile at least one of them is a $V U$; Horizontal Connector $(H C)$ is a $H U$ which connects at least two other distinct $H U$, meanwhile all of them belong to the same floor. More details of INSM can be found in (Liu and Zlatanova, 2012).

INSM, an intermediate model between the existing data and the desired logical model, provides advantages in two directions:

- It helps in further specification of semantics after a space is identified as NSC. As later discussed in section 4, we can investigate the geometric relationship between two test $N S C$ 's geometries (i.e. 'touch' or 'overlap' relation). If one of the NSC is $V U$, then the other NSC is a $V C$.

- INSM contains semantics that can facilitate path finding. For instance, if the start and the destination are on the same floor (i.e. the agent is moving horizontally), then $H C$ is dominated. In contrast, if a movement is between different floors, then priority will be given to $V C$.

\section{METHOD}

This paper presents our approach for automatic derivation of logical model from different existing building data with INSM. As a logical model can be automatically derived from INSM, this section mainly focuses on the construction of INSM from existing building data. 


\subsection{Prerequisites}

The conversion from existing building data to INSM data is completed under several assumptions and rules. We have to stipulate the semantic correspondence between original building data and related INSM data.

- Our method preserves original indoor structural division of buildings as much as possible. This means we do not subdivide large subspaces such as a lobby or long passages into smaller ones, unless there are vertical movement parts (e.g. walking steps) or obstacles to certain users. A subspace is determined as a node of logical model when a physical boundary (e.g. wall) between the spaces exists.

- In some special cases subspaces have no explicit connection, yet they are physically connected. Then the connection has to be given. For example, connection between stairs and landing places exists, yet there is no doors linking them. Thus we need to create a connection such as 'virtual opening'.

- We assume that connectivity and adjacency can be detected by geometry comparison if no explicit relationships are available in original building data. For instance, if there is an opening between two indoor subspaces, the geometric elements of the opening should either 'touch' or 'overlap' some elements of the two subspaces. Similarly, adjacency between two subspaces can be determined by 'touch' relations between the geometric elements of them. The 'touch' relation between two surfaces is confirmed with certain tolerance.

- If accessibility graph is required then the accessibility of subspaces should be provided by any other means. For example, supposing two rooms are connected via an opening yet the door cannot be open without key or can be open only in one direction (e.g. emergency exits)

- Semantics of the different subspaces in INSM is determined for original building data based on subspaces' definitions in INSM.

The main difficulty of automation is declaring the relations between subspaces. As mentioned above to support the conversion from original building data to INSM, some preprocessing of original data is required. Depending on the complexity and semantic richness of data, some information has to be revised. For example, walls, doors, windows, etc. might need to be simplified and represented by closed polygons. The section below presents the conversion procedure for existing building data.

\subsection{Workflow}

The workflow consists of four steps as shown in Fig 1. e approach is clearly geometry-based. Firstly, for each specific subspace we track the boundary and identify the geometric shapes; secondly, we add the necessary geometry or attributes; thirdly, all the subspaces are assigned corresponding semantics according to definitions of INSM; finally the logical network is derived.

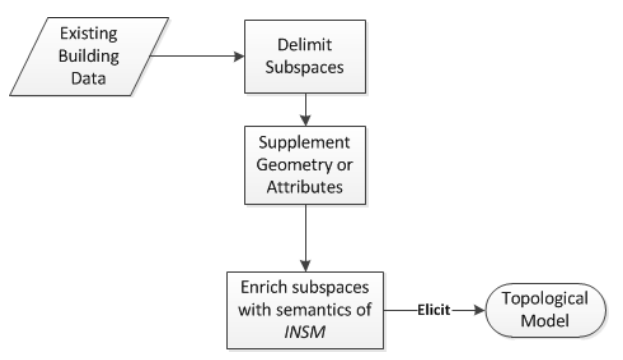

Figure 1. Workflow of generating topological model

The steps of the workflow are as follows:

1) Explicitly delimit $N S C$ and $O P N$; First of all, we have to conceptually know which part is a room/subspace. For instance, a corridor can be separated into several independent subspaces taking into account a partition because of steps. The subspaces correspond to NSC of INSM. After obtaining all NSC in a building, we can clarify $O P N$ between the NSC to indicate the connections of NSC. In most cases, OPN are physical doors. Normally this step is related to subdivision of indoor space, and sometimes manual work is required to add the virtual boundary of a subspace or indicate implicit connection between subspaces without physical bounds.

2) Attach corresponding geometry to the subspaces. After identification of NSC, the geometric representation of NSC should be determined. For example, a room can be represented by solid, 3D multiple surfaces or 2D polygon. Some special NSC are non-closed subspaces without physical boundary. Consequently $O P N$ on these non-closed NSC may need supplemental geometry (i.e. virtual $O P N$ ) or an indication of the connection between two NSC without explicit geometry (e.g. between a stair and a landing). In the previous case of the divided corridor, all the subspaces need virtual doors to indicate the connections. At this step manual work is needed for constructing the virtual geometry of some $O P N$.

3) Determine other specific semantics in INSM (i.e. $V U, V C$, $H C, E N D$ ). If a $N S C$ is determined and its geometry is provided, then the semantics of NSC will be automatically enriched based on geometric checking and definitions in INSM.

4) Check connectivity between subspaces by using openings and other information. Since the NSC and $O P N$ are learned from original data, we can build the relation of $N S C$-OPN-NSC according to the $N S C$ connected by $O P N$. Naturally the relation of NSC-NSC is known, which is just connectivity. With more information (e.g. a door needs key), we can determine the accessibility based on connectivity. Furthermore, according to geometric boundary we can check adjacency between NSC.

It might happen that two subspaces are connected by more than one openings. This information is also available in the INSM model by recording the number of openings attached to every subspace. Then this information is also propagated to the logical model. Each edge of the connectivity network may denote several openings between the two subspaces. Similarly, if all of 
the openings are not accessible to a specific user, then the edge will be not given as passable connection for the user.

\section{EXPERIMENT}

This section presents two experiments to demonstrate navigation model generation. The first one is based on semantic models, i.e. CityGML data; the second one is based on floor plans containing very limited semantics.

\subsection{CityGML}

Generally, the City GML LOD4 data contain information about rooms, walls, doors, windows, ceiling and floors. As the information is decoded by using GML syntax, it is easy to identify not only NSC but also relations ships such as belongs to/contains. A room consists of walls, ceiling and floor. A wall can contain doors and windows. If a specific CityGML file follows these rules, the information needed for INSM can be easily derived. Unfortunately, the available CityGML LOD4 datasets do not strictly follow the standard. In this test a CityGML LOD4 file was that is derived from an automatic procedure provided by Goetz, 2013.

The procedure consists of 1) mapping 2D poly gons (rooms) and points (doors) in JOSM editing tool, which is based on the photographs of floor plans; 2) setting up topological relationships of these geometric shapes and adding attributes (e.g. heights of rooms and floors, floor range of vertical passages); 3 ) registering the mapping result on OSM webserver to get unique object id; 4) building up a database to store the data; 5) running an automatic program to load data from the database and then reconstruct $3 \mathrm{D}$ building (e.g. extrusion on $2 \mathrm{D}$ room polygons); 6) outputting the data to CityGML format files.

We have created a 3D model of the OTB building according to the above procedure. Rooms/subspaces and doors/openings of the building are tagged respectively with 〈bldg.:interiorRoom > and <bldg.:opening > in the City GML document. However, this reconstruction procedure cannot generate indoor furniture (e.g. desks, chairs, coffee machines, etc.). It cannot provide exact details of staircase (e.g. steps, landing, railing, etc.) as well. In this manner, a staircase is created as a room. Thus, we can get relatively accurate geometry of regular rooms, doors and windows from the procedure. In order to distinguish stairs and elevators, we add explicit words 'stair' and 'elevator' in <gml:name> under the tag <bldg.:interiorRoom>.

In order to get connectivity network from the City GML file, we have to check the semantic relations between rooms and openings. Considering the unique id of each object (i.e. room and opening) it is straightforward to find the connection between rooms and openings. For instance, in Fig. 2 the tag <bldg:InteriorWallSurface > is before the tag 〈bldg:Door > and therefore the door attaches to the wall surface. Because the name of the surface includes the id of the room where it belongs, we can find the room to which the door relates. After we scan all the openings in the City GML file, the connected rooms of every opening are known. Topological relation (e.g. connectivity) can be built with such information.

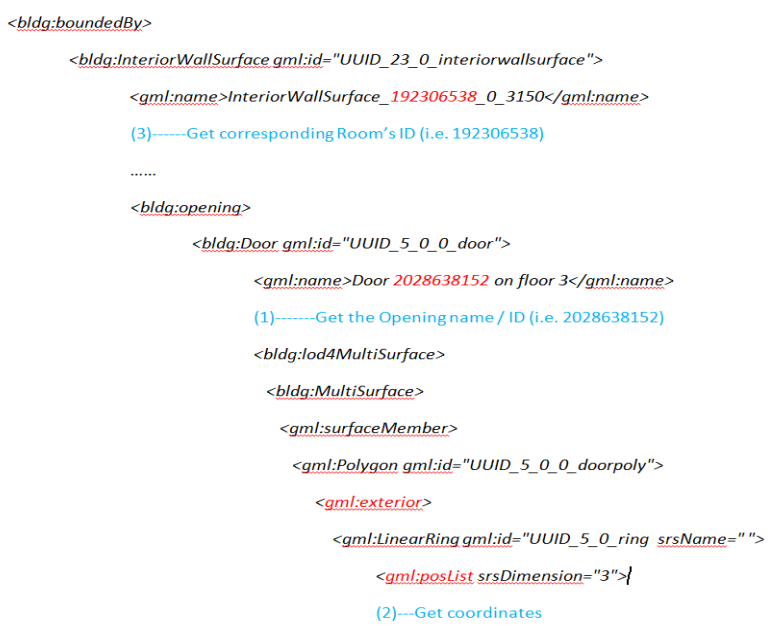

Figure 2. A fragment of the reconstructed City GML LOD4 file

Fig. 2 provides several key steps. The first step is to split the 'id' from a door's 'name'; the second step is to fetch the geometry of the door; the last step is to get the related 'Room' id of the door.

The following procedure describes how we derive a topological model from the reconstructed City GML file.

1. Get Opening's id, its Room id and the Opening's coordinates from the City GML file.

2. Get Room's name and id, and its geometry from the CityGML file. If a room's name contains "stair" or "elevator", then it is considered a vertical connection between two floors.

3. Maintain above information in $O P N$ and NSC classes.

4. Detect connectivity.

5. Enrich $N S C$ 's semantics (e.g. $V U, V C, H C$, etc.).

As it can be realised the CityGML file generated from the reconstruction procedure doesn't fulfil the structure indicated in the latest CityGML specification. Fig. 3 shows an LOD4 example dataset for a building in CityGML standard v2.0.0 (Gröger et al., 2012). In Fig. 3 there is a clear indentification of <bldg.:opening > beneath <bldg.:Room>, which indicates the attachment relation of a door to its room. In this manner, firstly we find a room; secondly we fetch the doors belonging to the room; the last step is to get the geometry of the room. Though Fig. 3 only displays the floor surface of the room, but actually we can get all the surface composing the room. 


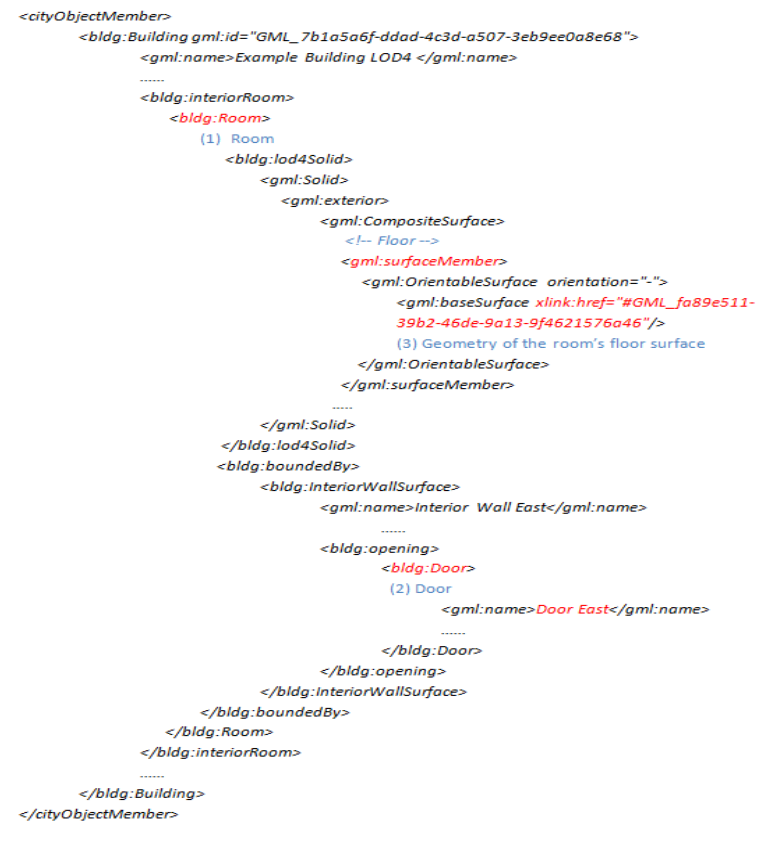

Figure 3. A standard City GML example dataset in LOD4

City GML provides a class "IntBuildingInstallation" to denote objects of either being associated with a Room, or with a complete building / building part (Gröger et al., 2012). For instance, interior stairs are typical examples of IntBuildingInstallation. In this manner we can get $V U$ information.

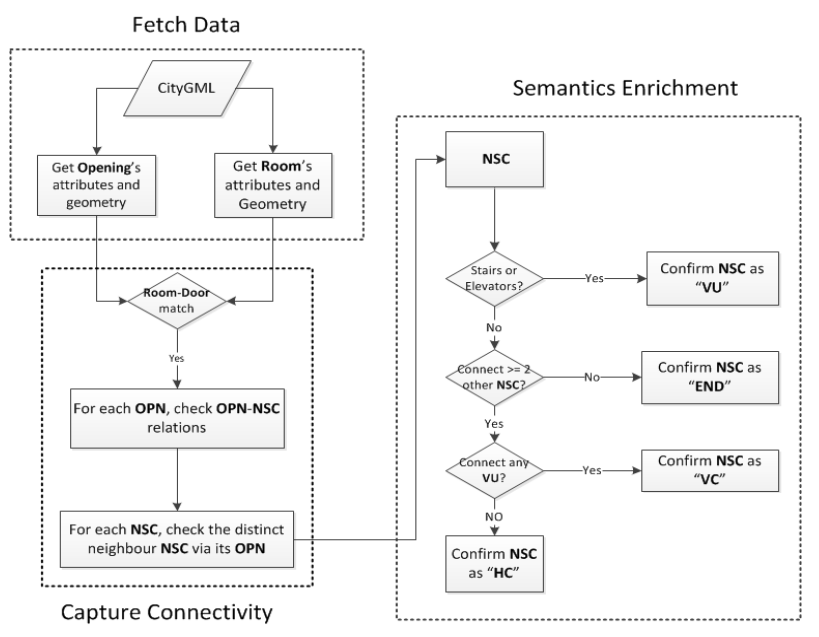

Figure 4. Flowchart on capturing connectivity and semantics enrichment

Figure 4 presents the workflow to load City GML data to main memory, capture connectivity and enrich semantics of rooms. The INSM is implemented in Oracle Spatial 11g. The workflow is completed in the memory by in-house software. After the processing all data are imported to the DBMS. Fig. 5 illustrates the City GML model of the OTB building and the corresponding connectivity network.

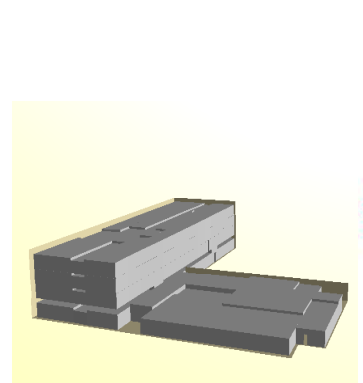

(a)

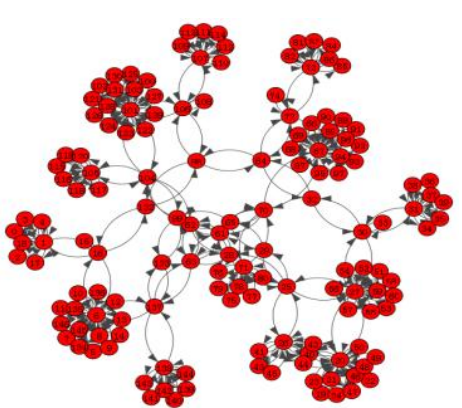

(b)

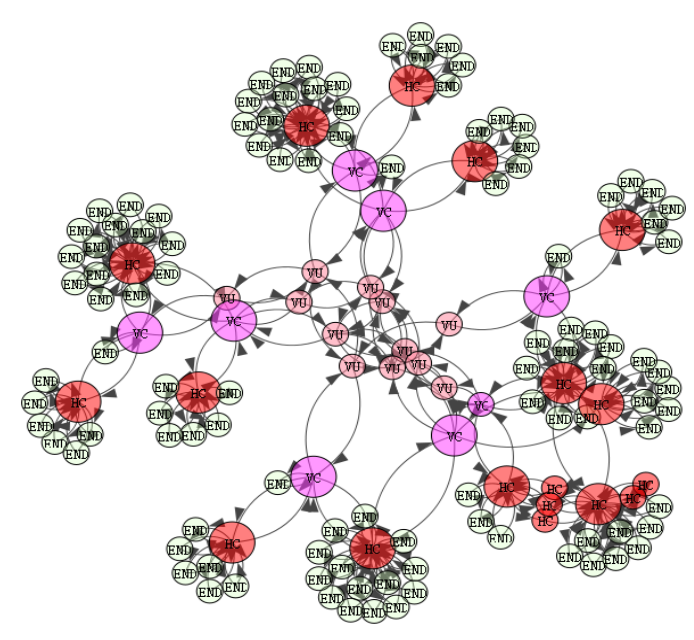

(c)

Figure 5: (a) The test building; (b) general connectivity network; (c) connectivity network enriched with INSM semantics

In the Fig. $5 \mathrm{~b}$ and Fig. $5 \mathrm{c}$, there are two edges between every two nodes, which mean the graph is a directional graph. We assume that initially two rooms are accessible from each other. By parsing CityGML we can directly obtain the rooms to which a door belongs. Then we count how many other distinct rooms are connected to the room via its openings (Fig. 4). Once we find all rooms connected with the room, each edge (related to certain opening(s) ) is spilt up to two directional edges. This is done to reflect accessibility constraints. In the current tests this information was not available therefore all the subspaces are always connected by two edges.

\subsection{Floor Plans}

The second procedure discusses creating INSM from floor plans. In this case all geometries are poly gons (including doors). If the polygons are not available, the floor plans have to be preprocessed. The connections between subspaces (polygons) are created by checking if neighbouring poly gons overlap or meet.

The floor plans used here are from the high-rise building at Delft named "Vermeer Toren" (Fig. 6a). For our experiments only three floors were used. As the floor configuration is identical, only one floor was created and copied at different heights. The floor plan was created by digitising manually the image of the floor plan in Bentley Systems. Only rooms (including stairs and elevator), corridors, and doors were created. The polygons 
representing rooms and corridors were stored in a layer named $N S C$ and doors were in a layer $O P N$. Several more doors were added for testing purposes. The connectivity per floor was created by checking the overlapping between the geometries in the floor.

Fig. $6 \mathrm{~b}$ demonstrates the reconstructed $2.5 \mathrm{D}$ models from the floor plans. The $V U$ of INSM, which denote stairs and one elevator, are represented by $3 \mathrm{D}$ polygons. These polygons were also included in the NSC layer. We automatically distinguish stairs and elevators by checking their slopes. Near 90 degree slope is the indication for an elevator, while bevel represents a stair. In addition, several virtual $O P N$ connecting $V U$ and $H U$ are created. Their connections are automatically detected by geometric overlap as well.

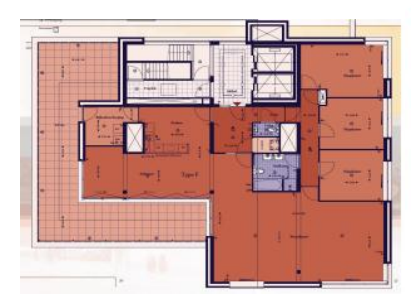

(a)

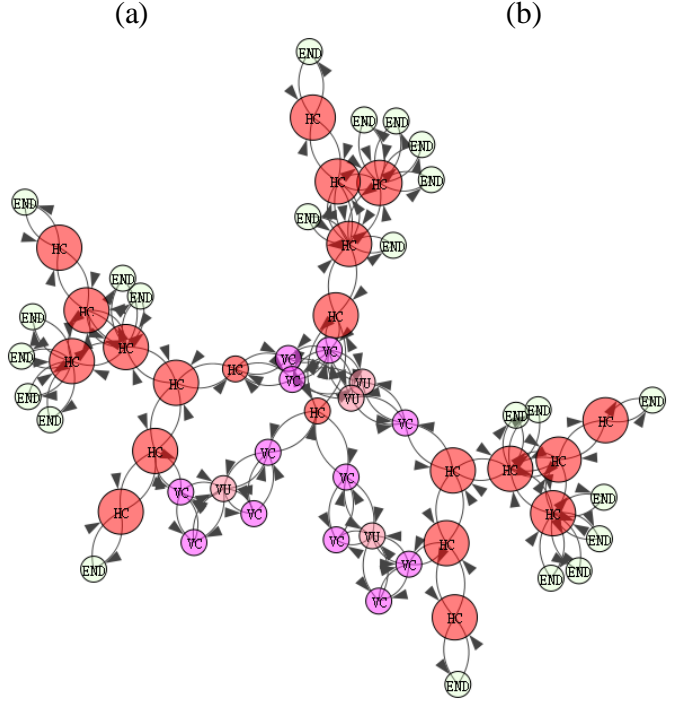

(c)

Figure 6. Vermeer building (a) original floor plans; (b) recreated 2.5D building model; and (c) connectivity network enriched with semantics

This test conveys the importance of semantics to generation of navigation models. Apparently there is seldom semantics in the original floor plans (Figure 6a). Basically they are pure geometric shapes. Thus in the manual reconstruction of $2.5 \mathrm{D}$ model we have introduced necessary semantics and rules (overlapping) to facilitate the generation of navigation model.

\section{ANALYS IS}

The above tests have clearly shown the difference between semantically rich and semantically poor data for populating INSM and extracting the connectivity network. In both cases the two main steps are: identifying navigable spaces and doors, and deriving the connectivity network.

Since our goal is to save efforts on manual work, it seems that semantic-rich data is a good option for identifying navigable spaces. Semantically rich data sets (CityGML and IFC) are generally straightforward to transform to INSM. However, CityGML lacks direct concepts for vertical building components such as elevators and stairs, which would require either manual interpretation or processing on attributes or geometry. The semantics can be stored either per building component or all components can be organised in one thematic layer.

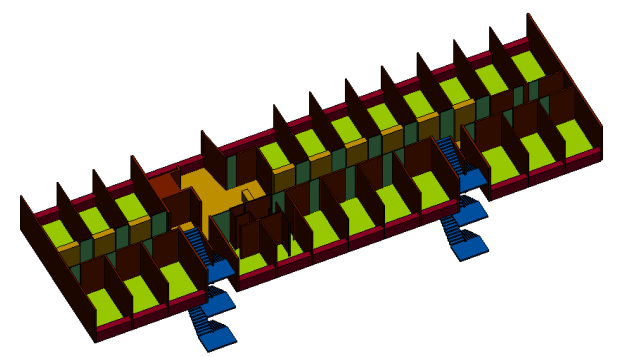

Figure 7. 3D model of OTB building

Datasets with little or no semantics at all (as the floor plan mentioned above) need additional human interpretation to identify spaces. The minimal semantics that has to be introduced is the concepts of NSC and $O P N$. The reconstructed model from floor plans can be used to derive topological models only after semantic enrichment.

There is major difference between semantically rich and semantically poor data on identification of connectivity relationships. In the case of CityGML the building components have explicit relationships ('part of', 'adjacent to'). Consequently the connectivity network can easily be extracted. Floor plans lack this information and the only option to detect relationship is geometric overlays. Apparently such overlays will be sensitive to the thickness of walls and doors. Yet the thickness is not considered for walls in our example.

To conclude, plenty of other data sets can be found, which have some kind of semantics but do not have the structure of the City GML. For example, the 3D floor in Fig.7 contains notations about semantics but it is organised by Layer. We cannot apply the criteria neither as in section 4.1 nor as in 4.2.

\section{OUTLOOK}

In this paper we have investigated the process of automatic creation of a connectivity network from existing data sets. We have performed tests with CityGML as the example of a semantically rich dataset and an image of a floor plan as the example of semantically poor dataset. Our goal is to create a semantically rich connectivity network. INSM was used as an example of semantically rich model that can automatically provide a connectivity network. 
The general conclusion is that semantically rich models are easier for navigation model generation compared to geometric models with limited semantics. However, the tests revealed many challenges which hinder the automatic procedures.

- The semantic models have different structures, which means that different types of relationships are explicitly recorded.

- The theoretical structure of the semantic models is not always followed. In some files topology can be only derived from the id of objects (section 4.1).

- The concepts for connections between floors are not explicitly provided in CityGML. Therefore further processing is needed of attributes or geometry.

If semantics is completely missing, the logical model for navigation will strongly depend on 3D geometric models or the reconstructed models of floor plans. In any case substantial preprocessing of the original geometry is needed (Dominguez et al 2012). During this pre-processing additional information (such as doors/windows) must be assigned to geometry to clarify and ensure the connectivity between spaces. In our experiments with floor plans we have provided semantics for rooms, vertical connections and doors. Nevertheless this semantics enrichment, we have no a standard way to derive navigation models from distinctly reconstructed models of floor plans.

Although the tests are rather limited, it is clear that semantic annotations are of critical importance for automatic network creation. The semantic annotations should be well-defined and standardised to facilitate easy exchange of navigation model.

\section{REFERENCES}

Becker, T., C. Nagel, T.H. Kolbe, 2009, A Multilayered SpaceEvent Model for Navigation in Indoor Spaces. In: 3D GeoInformation Sciences, Lecture Notes in Geoinformation and Cartography, 2009, Part II, 61-77.

Brown, G., C. Nagel, S. Zlatanova and T.H. Kolbe, 2013, Modelling 3D Topographic Space Against Indoor Navigation Requirements, In Progress and New Trends in 3D Geoinformation Science, LNG\&C, Springer, Heidelberg, New York, Dordrecht, London, pp. 1-22

Domınguez, B, A. Garcia, F. Feito, 2012, Semiautomatic detection of floor topology from cad architectural drawings, ComputerAided Design 44 (5) (2012) 367-378.

Goetz, M., 2013, Towards generating highly detailed 3D CityGML models from OpenStreetMap. International Journal of Geographical Information Science (IJGIS). Volume 27, Issue 5, pp. 845-865

Gröger, G., Kolbe, T. H., Nagel, C., and Häfele, K. 2012. OpenGIS City Geography Markup Language (CityGML) Encoding Standard v2.0.0. Open Geospatial Consortium Standard. Open Geospatial Consortium.

Lee, J., 2004, A spatial access-oriented implementation of a 3-D GIS topological data model for urban entities. Geoinformatica, 8 (3), pp. 237-264
Liu, L. and S. Zlatanova, 2012, A semantic data model for indoor navigation. In ISA '12 Workshop papers ACM SIGSPATIAL, pp. $1-8$

Lorenz, B., H.J. Ohlbach and E.P. Stoffel, 2006, A Hybrid Spatial Model for Representing Indoor Environments. In Proceedings of W2GIS (LNCS 4295) Hong Kong, China, pp. 102-112

Meijers, M., S. Zlatanova and N. Pfeifer, 2005, 3D geoinformation indoors: structuring for evacuation, In: Proceedings of Next generation 3D city models, 21-22 June, Bonn, Germany, 6 p.

$\mathrm{Pu}, \mathrm{S}$. and S. Zlatanova, 2005, Evacuation route calculation of inner buildings, In: Geo-information for disaster management, Springer Verlag, Heidelberg, pp. 1143-1161

Richter, K.-F., Winter, S. and Rüetschi, U.-J., 2009, Constructing Hierarchical Representations of Indoor Spaces. In: Tseng, Y.-C.; Scheuermann, P.; Güting, R. H. (Eds.), Tenth International Conference in Mobile Data Management, Workshop on Indoor Spatial Awareness. IEEE Press, Taipei, Taiwan, pp. 686-691.

Schaap, J.,S. Zlatanova and P.J.M. van Oosterom, 2012, Towards a 3D geo-data model to support pedestrian routing in multimodal public transport travel advices, In: Urban and Regional Data Management, UDMS Annual 2011, CRCpress/Taylor and Francis Group, Boca Raton, London, pp. 63-78

Stoffel, E., B. Lorenz and H. Ohlbach, 2007, Towards a Semantic Spatial Model for Pedestrian Indoor Navigation. In ER '07 Workshops, Advances in Conceptual Modeling, Springer, 328-337.

Thill, J.-C., T.H.D. Dao and Y. Zhou, 2011, Travelling in the three-dimecional city: applications in route planning, accessibility assessment. Location analysis and beyond, Journal of Transport Geography, 19 (12011), pp. 405-421

Yang, L. and Worboys, M.F. A navigation ontology for outdoorindoor space. Third ACM SIGSPATIAL International Workshop on Indoor Spatial Awareness (ISA 2011), November, Chicago, IL. 2011.

Winter, S., 2012, Indoor Spatial Information. International Journal of 3D Information Modelling, 1 (1): 25-42. 